Besonders auffällig ist die Verteilung der Triploiden auf die einzelnen Kulturen (Tab. 3). Ohne weiteren statistischen Vergleich ist es klar, daß solche Verteilungen, wie sie besonders bei $0^{0} 2$ Stdn. und bei $-4^{0} 1$ Std., aber auch bei den anderen Versuchsgruppen mit mehr als einem $3 n-q$ beobachtet wurden, nicht eine Zufallsverteilung wiedergeben ${ }^{6}$. Sie zeigen vielmehr eine Häufung der Triploiden in den Einzelkulturen an. Die Erklärung dafür ist nach dem oben Gesagten offensichtlich; während das Auftreten der Triploiden in den Kontrollversuchen wohl allein auf meiotische Störungen zurückgeht, müssen einzelne der behandelten $f q$ als Folge der Polyploidisierung der Urkeimzellen tetraploide Ovarteile, einzelne

6 Unter der vereinfachenden Annahme, daß die Einzelkulturen gleiche Individuenanzahlen aufwiesen, berechnet sich die Wahrscheinlichkeit dafiir, daß die Gesamtverteilung der Triploiden in den Kälteversuchskulturen (Tab. 3, unterste Zeile) einer $\mathrm{P}$ o i s s on-Verteilung entspricht, a uf $\mathrm{P}<10-6$. oder Gruppen von Eiröhren, besitzen, aus denen mehrere diploide Eizellen entstehen.

Daß nur so wenige größere Gruppen von Triploiden beobachtet wurden, liegt zum Teil daran, daß nur ${ }^{1 / 3}$ der diploiden Eizellen erfaßt werden; denn nicht nur ${ }^{1 / 3}$ der $3 n-q+$ entzieht sich der Beobachtung oder stirbt ab, sondern auch eine den 3n-Zygoten gleiche Zahl von XXY-Zygoten bleibt unberïcksichtigt. Außerdem werden auch wohl nicht wenige hypo- oder hyperdiploide Eizellen entstehen. Die Beschränkung der Triploidenzeugung auf nur vereinzelte $f f$ schließlich liegt zweifellos daran, daß nur in einem geringen Teil der behandelten Eier einzelne Urkeimzellen gerade in dem für die Restitutions kernbildung geeigneten Mitosestadium sind. Es ist wahrscheinlich, daß eine mehrfache Kältebehandlung mit kurzen Erwärmungspausen einen noch erheblich günstigeren Versuchsausfall gibt.

Hrn. G. W i e r t z, der die Versuche ausgeführt hat, bin ich für seine Hilfe dankbar.

\title{
Unregelmäßige Chromosomenverteilung durch eine Mutation bei Ephestia kühniella Z.
}

\author{
Von Alfred Kühn und Dore-Li Woywod \\ Aus dem Kaiser-Wilhelm-Institut für Biologie, Hechingen \\ (Z. Naturforschg. 1, 38-44 [1946]; eingegangen am 1. September 1945)
}

\begin{abstract}
Die unvollständig dominante Mutation Mo bewirkt unregelmäßige Chromosomenverteilung, meist in einer der ersten embryonalen Teilungen. In dem reinen Stamm entstehen etwa 20\% Mosaiktiere, meist Halbseitenmosaiks, die verschieden helle Flügelschuppen haben. Im Mittel ist die eine Seite gegenüber der Flügelhelligkeit der gleichseitigen Tiere des Stammes aufgehellt, die andere verdunkelt. Nach Einkreuzung spalten verschiedene rezessive mutierte Allele nicht gekoppelter Gene mosaikhaft heraus. Mo bewirkt also die unregelmäßige Verteilung verschiedener Chromosomen. Das auf der einen Seite ausfallende Chromosom kann ganz verloren gehen oder der anderen Seite zugeteilt werden. Auch zweifach monosome Flügel können entstehen.
\end{abstract}

$\mathrm{I}_{\mathrm{u}}^{\mathrm{n}}$ n unseren Zuchten von Ephestia kühniella und von Ptychopoda seriata kommen ab und zu Mosaiktiere vor. Drei Fälle wurden früher schon beschrieben: von Ephestia ein Sektorialmosaik $^{1}$ und ein Halbseitenmosaik ${ }^{2}$, in

1 A. K ühn, Versuche üher die Wirkungsweise der Erbanlagen. Naturwiss. 24, 6 | 1936].

2 A. K ühn, Zur Entwicklungsphysiologie der Schmetterlingsschuppen. Biol. Zhl. 61, 136 ff. [1941]. denen bei einem $+\mid b$-Heterozygoten eine Seite $b$ zeigte, und von Plychopoda ein Halbseitenmosaik ${ }^{3}$, bei dem in einer wildfarbigen Zucht auf einer Seite die der Mutation At entsprechende Flïgelbeschuppung erschien.

${ }^{3}$ A. K ü h n u. M. v. Eng elh a r d t, Uber zwei melanistische Mutationen (At und $n i$ ) von Ptychopoda seriata Schrk. Biol. Zhl. 63, 264 ff. [1943]. 
In einem Ephestia-Stamm, der $d z^{4}$ enthält, treten seit 1943 regelmäßig ung. 20\% Mosaiktiere auf (Tab.1), in der Regel Halbseitenmosaiks, bei denen die Flügel der einen Seite viel hellere Schuppen tragen als die der anderen (Abb. 1). Beide Geschlechter sind gleich oft mit der Eigenschaft behaftet ( $ㅇ$ 20,0\%, $\left.0^{7} \sigma^{7} 19,7 \%\right)$. Die Erscheinung wird gleichermaßen von Mosaiktieren wie von normal aussehenden Geschwistern übertragen. Die Kreuzungen mit verschiedenen anderen Stämmen zeigen die monofaktorielle Erblichkeit der Er-

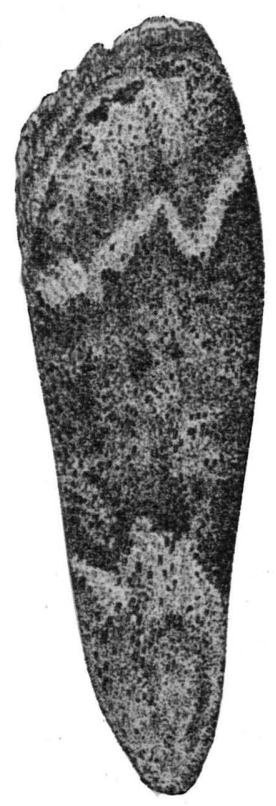

a

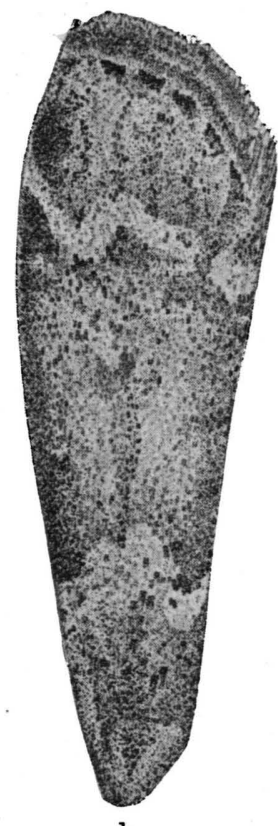

b
Abb. 1. Vorderflügel eines Halbseitenmosaiks aus dem Mo-Stamm. 8/1 (F'reiberg gez.).

\begin{tabular}{|c|c|c|}
\hline & $\%$ Mosaiks $( \pm m)$ & $\mathrm{n}$ \\
\hline Mo-Stamm . . . . & $19,8( \pm 0,8)$ & 2404 \\
\hline $\mathrm{F}_{1} \ldots \ldots \ldots$ & $4,0( \pm 0,3)$ & 3410 \\
\hline $\mathrm{F}_{2} \ldots \ldots$ & $\begin{array}{l}\quad 8,9( \pm 0,7) \\
\text { Erw. } 7,0\end{array}$ & 1874 \\
\hline $\mathrm{R}: \mathrm{F}_{1} \times \mathrm{Mo}_{0} \ldots$ & $\begin{array}{l}14,6( \pm 2,6) \\
\text { Erw. 11,9 }\end{array}$ & 198 \\
\hline
\end{tabular}

Tab. 1. Häufigkeit von Mosaiktieren in dem Mo-Stamm und in Kreuzungen.

4 A. Küh n, Über eine geschlechtsgekoppelte Mutation $(d z)$ bei Ephestia kühniella Z. Biol. Zbl. 59 [1939]. scheinung (Tab. 1). Sie ist unvollständig dominant; die Penetranz in $F_{1}$ beträgt $4 \%$. In $F_{2}$ und der Rückkreuzung mit dem Mosaikstamm liegt die Anzahl der Mosaiktiere etwas über der Erwartung für monohybriden Erbgang. Das
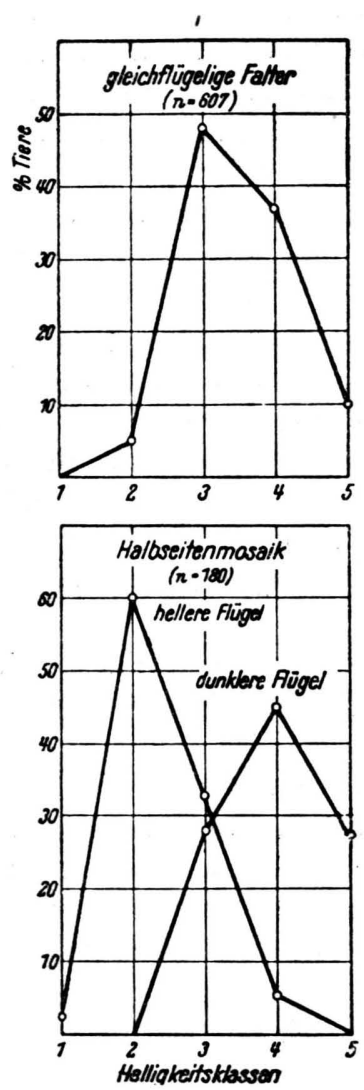

Abb. 2. Variantenverteilung in der Helligkeitsskala bei gleichflügeligen Faltern und Halbseitenmosaiks in dem Mo-Stamm.

mutierte Allel, welches mit einer bestimmten Penetranz die Mosaikausbildung hervorruft, nennen wir Mo (Mosaik).

Die Wirkung von Mo tritt während der Ausfärbung der Flügel in der Puppe hervor. Bei Halbseitenmosaiks steigt der Pigmentierungsgrad der Puppenflügel auf der einen Körperseite langsamer an und bleibt bei einem tieferen Werte stehen als auf der anderen. Der Helligkeitsunterschied $\mathbf{z w i s c h e n ~ d e n ~ F a l t e r f l u ̈ g e l n ~}$ der beiden Seiten ist verschieden groß. Um ein Maß für ihn zu gewinnen, wurden die Flügel mit einer Skala gemessen, die durch 4 Test- 
flügel in 5 Klassen eingeteilt ist. Ein Vergleich der Variantenverteilung der Flügel der Halbseitenmosaiktiere mit der der gleichflügeligen Tiere des Mosaikstammes (Abb.2) zeigt, daß der hellere Flügel $(M \pm m=2,41 \pm 0,05)$ im Mittel um etwa 1 Klassenwert gegenüber der Norm der Gleichflügeligen $(\mathrm{M} \pm \mathrm{m}=3,51 \pm$ $0,01)$ aufgehellt ist. Bei $62,2 \%$ fallen die helleren Flügel in die Klassen 1 und 2 der Helligkeitsskala, während von den beiderseits gleichhellen Tieren des Stammes nur 5,3\% die Klasse 2 erreichen. Die dunkleren Flügel der Halbseitentiere stimmen im Mittel auch nicht mit den Gleichflügeligen überein; ihr Mittelwert ( $\mathrm{M} \pm$ $\mathrm{m}=4,01 \pm 0,06)$ ist um einen geringeren Betrag als der der helleren Flügel gegenüber dem Mittelwert der Norm nach der dunkleren Seite der Skala verschoben. Also steht bei einem Teil der Mosaiktiere der Aufhellung der einen Seite eine Verdunkelung der anderen Seite gegenüber.

Ein Wechsel der Flügelhelligkeit kann bei Ephestia auf verschiedene Weise zustandekommen. Das Zeichnungsmuster der Wildform setzt sich aus verschieden stark pigmentierten Schuppen, dunklen Zeichnungsschuppen, hellen Zeichnungsschuppen und dazwischenstehenden Untergrundschuppen, zusammen (Abb. 1a, 7a). Diese Schuppentypen unterscheiden sich nicht nur durch die Einlagerung verschiedener Pigmentmengen, sondern auch in ihrer Chitinstruktur und ihrer Form $5,2$. Bei einer Reihe von verschieden hellen Ephestia-Stämmen wechseln die Helligkeit des Untergrundes und die Dunkelheit und Ausdehnung der Zeichnung, und dabei wechselt jeweils mit der Pigmentierung der Schuppen auch der Struktur- und Formtypus der Schuppen (1.c. ${ }^{5}$, S. 165 ff.). Bei der Mutation $d z$ wird der Untergrund des Zentralfeldes von schlanken dunklen Zeichnungsschuppen durchsetzt (Abb.1a). Bei der schwarzen Rasse (b) wird der ganze Untergrund von dunklen Zeichnungsschuppen eingenommen, und nur die weißen Querbinden tre-

5 A. Kühn u. K. Henke, Genetische und entwicklungsphysiologische Untersuchungen an der Mehlmotte Ephestia kühniella Zeller. VIII-XII. Abh. Ges. Wiss. Göttingen, Math.-physik. Kl. 15 [1932]. ten als Zeichnungssystem hervor. Die Mutation $m l^{6}$ (und 1. c. ${ }^{2}$, S. 140 ff.) erlaubt überhaupt nur die Ausbildung heller Zeichnungsschuppen mit breitem weißen Rand und macht dadurch den Flügel musterlos. Diesen Mutationen stehen andere gegenüber, bei denen die Verteilung von Struktur-Form-Typen der Schuppen über den Flügel nicht abgeändert wird, jedoch die Einlagerung von Pigment in jedem Schuppentypus herabgesetzt $(h e)^{7}$ oder erhöht wird $(v d)^{8}$. Die Aufhellung und Verdunkelung der Flügel bei der Wirkung von Mo kommt auf die zweite Weise zustande. Das zeigt sich besonders deutlich bei schwarzen $(b / b-)$ und musterlosen ( $\mathrm{ml} / \mathrm{ml}$-) Tieren, welche nach Kreuzung mit dem $M o$-Stamm in $\mathrm{F}_{\mathbf{2}}$ herausspalten. Wenn $M o$ bei $b / b$ sich ausprägt, ist der eine Flügel gleichmäßig zu grau-braun aufgehellt, aber alle Schuppen haben die typische Gestalt der dunklen Zeichnungsschuppen. Auf den Flügeln von $\mathrm{ml} / \mathrm{ml}$-Tieren, in denen $M o$ sich manifestiert, stehen typische helle Zeichnungsschuppen; die Aufhellung des einen Flügels kommt dadurch zustande, daß der unterhalb des weißen Randes liegende pigmentierte Schuppenteil gegenüber den typischen Schuppen dieses Struktur-Form-Typus verblaßt ist. Bei einzelnen Mo-ml/ml-Tieren aus $\mathrm{F}_{2}$ ist auf der Gegenseite der Schuppenschaft wesentlich dunkler als bei der Norm, und das Pigment dringt weiter gegen das Schuppenende hin vor (Abb.3). Bei der mosaikhaften Aufhellung und Verdunkelung infolge der Mo-Wirkung wird also nicht der Struktur-Form-Typus der Schuppen, sondern nur die Pigmenteinlagerung abgeändert.

Solche Mosaikbildungen weisen mit großer Wahrscheinlichkeit auf eine Elimination eines Chromosoms hin, die bei einem Halbseitenmosaik in einer der ersten Furchungsteilungen auftreten muß. Von Drosophila ist ein Fall be-

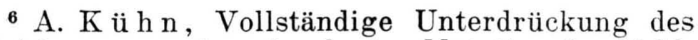
Zeichnungsmusters durch eine Mutation der Mehlmotte Ephestia kühniella Z. Naturwiss. 27 [1939].

$7 \mathrm{~A} . \mathrm{K} \ddot{\mathrm{uhn}} \mathrm{n}$, Úber die Mutation he (gehemmte Ausfärbung) bei Ephestia kühniella Z. Z. Vererbgsl. 77 [1939].

${ }_{8} \mathrm{~A} . \mathrm{K} \ddot{\mathrm{u} h \mathrm{n}}$, Über eine die Schuppenformbildung und die Schuppenpigmentierung beeinflussende Mutation (vd) von Ephestia kühniella Z. Biol. Zbl. 64 [1944]. 
kannt, in dem eine Mutation die somatische Elimination von Chromosomen bewirkt ${ }^{9}$. Eine cytologische Bestätigung des Chromosomenverlusts wie bei Drosophila wird bei Ephestia mit ihren zahlreichen kleinen Chromosomen ( 2 n bei $\& \&=59$, bei $\left.\sigma^{x} \sigma^{x}=60\right)$ sich schwerlich erbringen lassen; doch läft er sich aus bestimmten Mosaikbildungen mit Sicherheit erschließen.

Wenn der Helligkeitsunterschied der Fliigel der Halbseitenmosaiks des Mo-Stammes (Abb.1) auf einer unregelmäßigen Chromosomenverteilung beruht, so muß auf der einen Seite des Keimes ein Chromosom ausgefallen sein, das einen Faktor enthält, der die Schuppenpigmentierung beeinflußt und zwar so, daß die Allele sich in der Wirliung addieren. Für das auf der einen Seite ausfallende Chromosom sind zwei Möglichkeiten gegeben: Entweder kann es bei der unregelmäßig verlaufenden Teilung ganz verloren gehen (Abb. 4 a), oder es kann mit der anderen Spalthälfte abriicken (Abb. 4b), so daß einerseits monosome, andererseits trisome Körperteile entstehen. Daß diese zweite Yöglichkeit mindestens in einem Teile der Fälle verwirklicht ist, wird dadurch sehr wahrscheinlich gemacht, daß bei den Halbseitenmosaiks im Mittel beide Flïgel in entgegengesetztem Sinne von der Norm abweichen. Wir bezeichnen den die Pigmentierung der Schuppen beeinflussenden, nicht durch eine Genmutation im Kreuzunosexperiment erfaften, sondern aus der Mosaikbildung erschlossenen Faktor mit $\mathrm{Pm}$. Ob er die Pigmenteinlagerung in die Schuppen steigert, der Verlust eines Allels also aufhellend, Hinzufügung eines dritten Allals verdunkelnd wirkt, oder ob Pm die Pigmentierung drosselt und die Monosomie sie deshalb höher steigen läßt, und Trisomie sie mehr herabdrïckt, ist nach Halbseiten-Helligkeitsmosaiks nicht zu entscheiden. Da die Variationskurve der aufgehellten und der verdunkelten Flügel mit der Norm transgrediert, läßst sich in keinem Einzelfall mit Sicherheit sagen, ob nur ein Flügel verändert ist. Aber eine sektorielle Aufhellung in einem normalen Muster, wie sie in einem Fall beob-

9 A. II. Sturtevant, The claret mutant type of Drosophila simulans: a study of chromosome elimination and of cell-lineage. Z. wiss. Zool. 135 [1929]. achtet wurde, ist nur durch einseitigen Chromosomenausfall (Abb.4a) zu erklären, und daraus ergibt sich der Schluß, daß der Chromosomenverlust aufhellend wirkt, $P m$ also ein die Schuppenpigmentierung steigernder Faktor ist. Unter den $b / b$-Tieren aus $\mathrm{F}_{2}$ und Nachzuchten aus Kreuzungen des Mo-Stammes mit Schwarzschuppigen, bei denen der Pigmentierungsgrad der aufgehellten Flïgel nicht mit der Norm

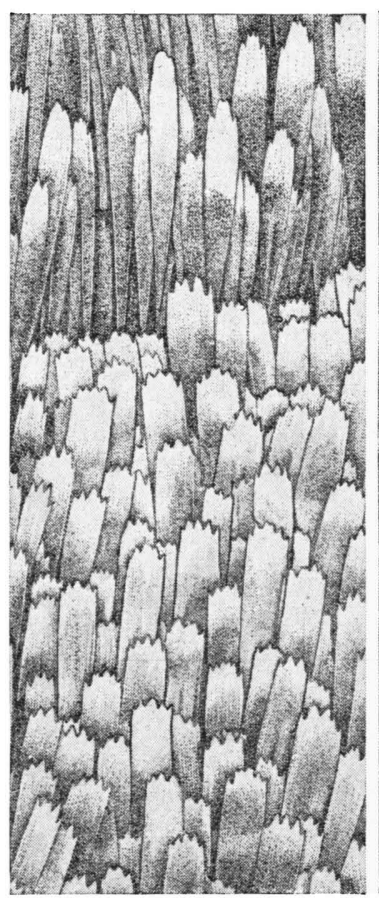

a

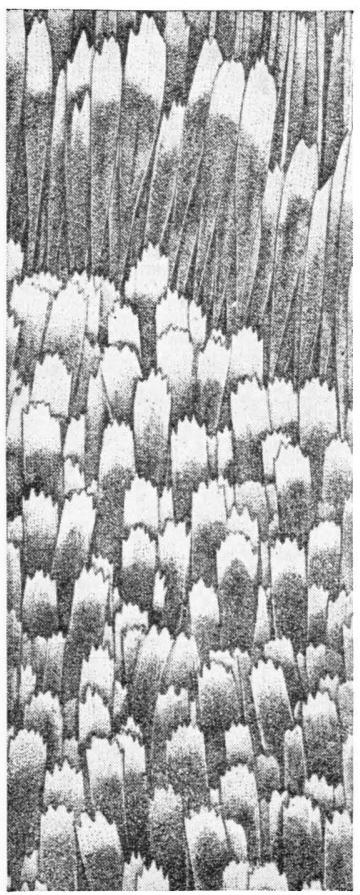

b
Abb. 3. Einander entsprechende Stücke rom Flügelaußenrand der heiden Flügel eines $\mathrm{ml} / \mathrm{ml}$-Iflligkeitsmosaiks aus $F_{2}$ nach Einkreuzung von $m l$ in den Mo-Stamm. 70/1 (Freiberg gez.).

transgrediert, kommen einzelne vor, die auf $b e i$ den Seiten aufgehellt sind. Sie können dadurch entstehen, daß bei einer frühembryonalenTeilung beide Spalthälften des einen den Faktor Pm enthaltenden Chromosoms (Abb.4c) oder je eine Spalthälfte der beiden $\mathrm{Pm}$-Chromosomen auf entgegengesetzten Seiten (Abb. 4 d) verloren gehen.

Daß Pm mit dem Faktor Mo, der die unregelmïßige Chromosomenverteilung auslöst, identisch ist, ist sehr unwahrscheinlich; Mo/Mound t/Mo-Tiere ohne Mosaikbildung sind gleich wie $+1+$-Tiere pigmentiert. Vielleicht 

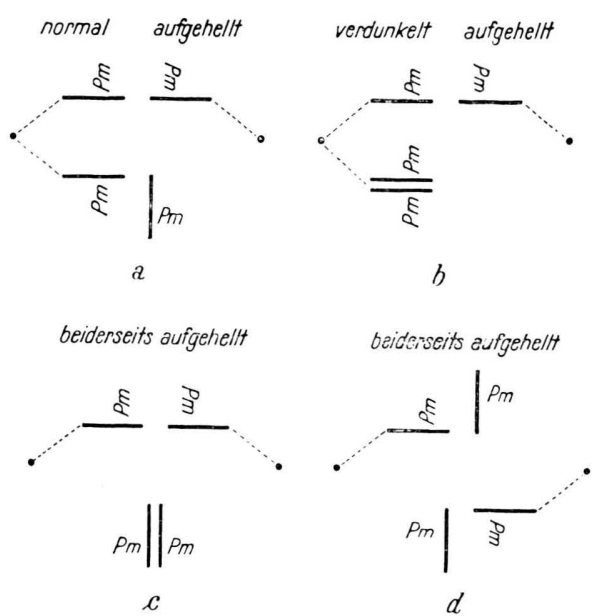

Abb. 4. Schema der unregelmäßigen Chromosomenverteilung in dem Mo-Stamm.

ist Pm mit Mo gekoppelt; aber es ist auch möglich, daß Mo und Pm in verschiedenen Chromosomen liegen und die Häufigkeit des Auftretens eines Helligkeitsmosaiks die Empfindlichkeit des $\mathrm{Pm}$-Chromosoms für die Mo-Wirkung anzeigt.

Daß Mo nicht nur auf die Verteilung der Spalthälften desjenigen Chromosoms wirkt, in dem das mutierte Gen liegt, sondernauch auf die Verteilung anderer Chromosomen, ebenso wie die Mutation claret von Drosophila ${ }^{9}$, beweist das mosaikhafte Auftreten eingekreuzter rezessiver Gene in $\mathrm{F}_{1}$ und $\mathrm{F}_{2}$ der Mo-Kreuzungen. Durch sie wird die chromosomale Interpretation, die für die Helligkeitsmosaiks nur sehr wahrscheinlich ist, bewiesen.

In einem Fall war die rechte Hälfte eines $+\mid$ Mo $+\mid m l d z / d z-o^{7}$ musterlos, und da sich die hellen Zeichnungsschuppen sehr deutlich ron den viel dunkleren Schuppen abheben, die normalerweise auf dem Körper des Falters stehen, ließ sich die Trennungslinie auch über Kopf, Thorax und Abdomen verfolgen. Sie verlief auf dem Thorax ziemlich genau median, auf dem Kopf und dem Abdomen wich sie etwas nach rechts ab. Die Beine und die Antenne der rechten Seite waren hell wie die Flügel.

Besonders disponiert für die unregelmäßige Verteilung ist offenbar das $b$-Chromosom; denn das Herausspalten von $b$ in $+/ b$-Tieren, halbseitig oder sektorial, ist vereinzelt auch in anderen Stämmen schon aufgetreten. Stets sind dabei die Schuppen auf der $b$-Seite nicht nur schwarz wie bei $b / b$-Tieren, sondern auch stark verkleinert (Abb.5b). Bei einem Mosaiktier, dessen Schuppen im Abklatschpräparat vermessen wurden, sind sie im Mittel um etwa $1 / 5$ verkürzt und um die Hälfte verschmälert ${ }^{2}$. Bei allen $+\mid b$-Mosaiks waren auch die Flügel auf der $b$-Seite etwas kleiner als auf der wildfar,bigen Seite. Da $b$ selbst nicht mit einer Schuppen- und Flügelverkleinerung verknüpft ist, muß diese auf einer anderen Wirkung des Chromosomenverlusts beruhen. Aber nicht jeder Chro-

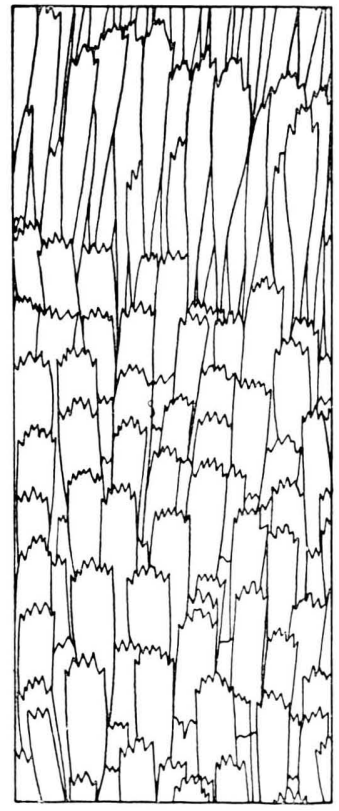

a

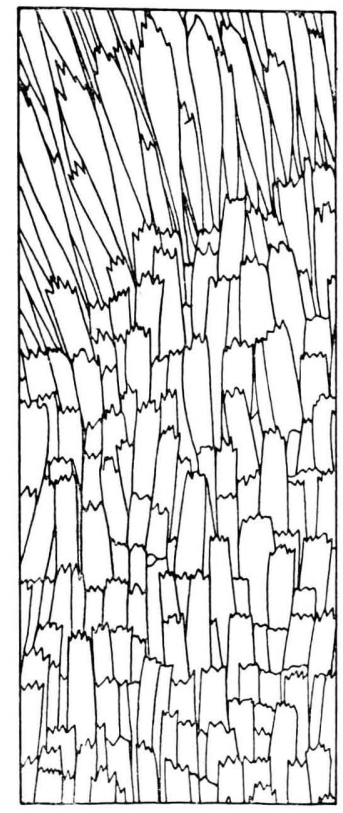

b

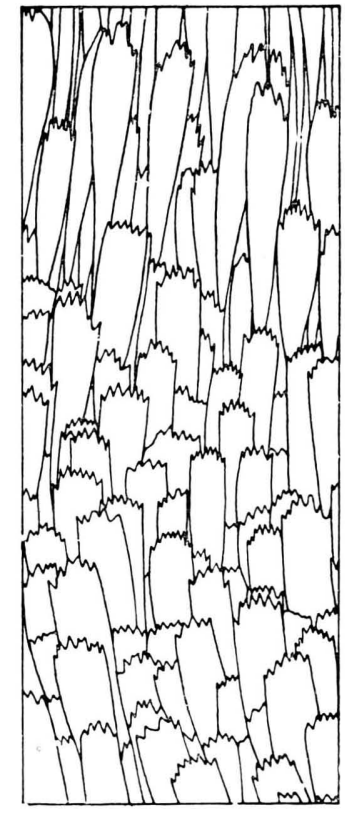

Abb. 5. Schuppenumrisse auf einander entsprechenden Vorderflügelstücken: a normal wildfarbig, b schwarzschuppiger Flügel $(b)$ eines Halbseitenmosails, c wildfarbiger Flügel $(b /+/+)$ desselben Halbseitenmosaiks. $70 / 1$ (F reiberg gez.). 
mosomenverlust wirkt auf das Schuppen- und Flïgelwachstum; bei dem $+\mid m l$-Mosaik sind, wie bei den Helligkeitsmosaiks, keine Größenunterschiede zu bemerken. In dem $b$-Chromosom muß also ein die Schuppengröße beeinflußender Faktor liegen; wir nennen ihn $S g$. Das wird auch bestätigt durch die Schuppenausbildung auf der wildfarbigen Seite: Hier sind die Schuppen entweder von normaler Größe, wie bei dem $1941^{2}$ beschriebenen Tier, oder sie sind bedeutend vergrößert (Abb.5c). Die beiden Fälle werden durch das Schema Abb. 6 erklärt.

Manchmal betrifft der Unterschied zwischen den Flügeln von Halbseitentieren in dem MoStamm und Kreuzungen auch andere Merkmale als die Schuppenhelligkeit und die Manifesta-

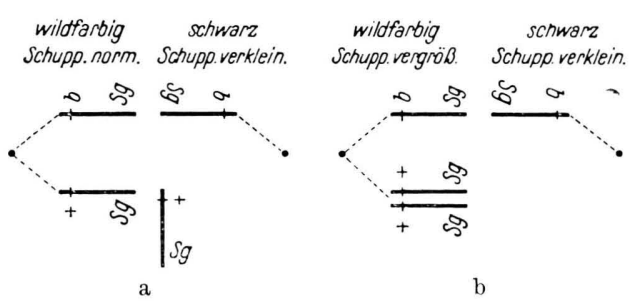

1bł. 6. Schema der Chromosomenverteilung bei den Mosaikbildungen in $\underset{M o}{+} \frac{+}{b}$.

tion bekannter rezessiver Allele in Heterozygoten. So zeigte sich einmal auf dem linken Flügel bei einem $\& \mathrm{Mo} / \mathrm{Modz}$, eine sehr starke Zusammenschiebung der Querbinden bis $\mathrm{zu}$ fast völligem Schwund des Zentralfeldes, wie sie der Genkombination +/Sydz entspricht. Da $S y$ dominant ist (homozygot letal ${ }^{10,11}$ ), d. h. $1 S y^{+}$ eine geringere Ausbreitung des Symmetriefeldes ${ }^{12}$ bewirkt als $2 \mathrm{Sy}^{+}$, so ist es möglich, daß diese Mosaikbildung auf dem Ausfall eines Sy+-Chromosoms auf der einen Seite beruht, analog wie ein Chromosomenausfall bei Pty-

10 A. K ̈̈hn, Entwicklungsphysiologische Wirkungen einiger Gene von Ephestia kühniella. Naturwiss. 20 [1932].

${ }^{11}$ A. K ühn u. K. Henke, Genetische und entwicklungsphysiologische Untersuchungen an der Nehlmotte Ephestia kühniella Zeller. XIII. u. XIV. Abh. Ges. Wiss. Göttingen, Math.-phys. Kl. 15 [1936].

12 I. K i h n u. M. v. Engelh a r d t. Ther die Determination des Srmmetriesrstems auf dem Vorderfliigel von Elhestia kïhniella Zeller. Roux' Arch. $130[1933]$. chopoda den Phänotypus von At mosaikhaft hervortreten läßt.

Bei einem Bastard- $f+/ M o+/ m l d z$ ist der eine Flügel musterlos und hochgradig aufgehellt (Abb. 7). Diese Mosaikbildung kann nur darauf beruhen, daß auf der abnormen Seite

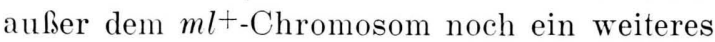
Chromosom mit einem auf die Schuppenhelligkeit wirkenden Faktor ausgefallen ist, wahr-

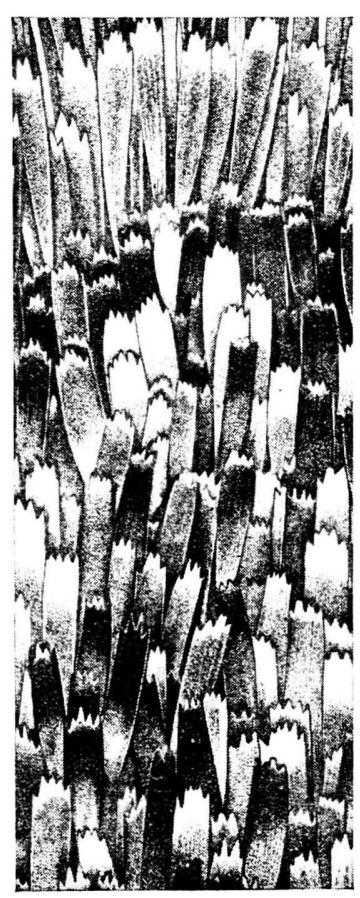

a

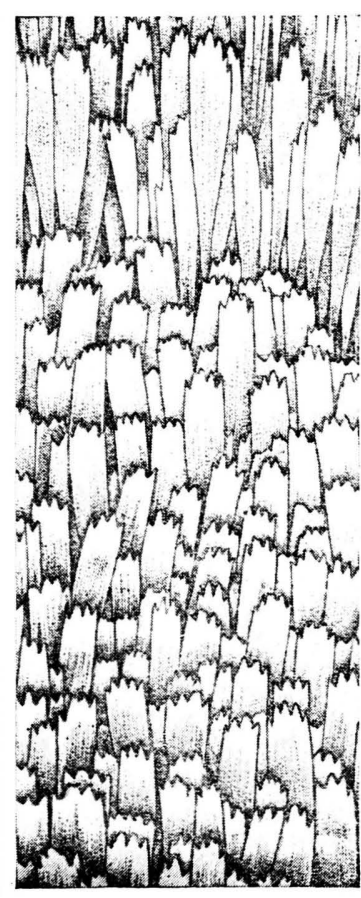

b
Abb. 7. Einander entsprechende Stücke eines Halbseitenmosaiks des Ausgangsgenotypus $\frac{+}{M o} \frac{t}{m l}$; rechts $m l$ und aufgehellt $\left(\frac{-}{m l} \frac{-}{P m}\right)^{70 / 1}$ (Freiberg gez.).

scheinlich dasselbe, welches auch in dem $\mathrm{Mo}$ Stamm und in $\mathrm{F}_{1}$ und $\mathrm{F}_{2}$ bei wildfarbigen, $\mathrm{ml} / \mathrm{ml}$ und $b / b$-Tieren durch seinen Verlust die Aufhellung bewirkt. Der abnorme Flügel ist nicht kleiner als der andere. Bei Ephestia (mit 29 Autosomen im Genom) sind also auch bestimmte zweifach monosome somatische Zellen entwicklungsłähig, während bei Drosophila, außer der Elimination eines der beiden X-Chromosomen, nur der Ausfall des einen kleinen IV. Chromosoms ertragen wird, während der Verlust eines der beiden großen Autosomen zell-letal wirkt. 
Die Mosaiktiere des Mo-Stammes und seiner Kreuzungen sind häufig steril. Während von den Paarungen normal aussehender Geschwister nur etwa ${ }^{1} / 10$ erfolglos ist, bleibt ungefähr die Hälfte der Paarungen, in denen beide Partner oder die Weibchen Mosaiks sind, und etwa 1/3 der Paarungen mit einem Mosaikmännchen ohne Gelege. Gynander wurden bisher nicht beobachtet. Die Gelegegröße, welche in dem Mo-Stamm im Verhältnis zu anderen Inzuchtstämmen überhaupt gering ist, ist bei den fruchtbaren Helligkeitsmosaik- $f$ noch um etwa
$30 \%$ kleiner als bei den normalaussehenden Geschwister- + \&. Auch die Aufwachszahl der Nachkommen von Mosaikeltern ist niederer als in Zuchten, bei deren Eltern Mo sich nicht ausprägte. Die Nachkommenzahl in Zuchten mit einem oder zwei Mosaikeltern ist im Mittel nur etwa halb so groß wie in gleichzeitig laufenden Zuchten von Nicht-Mosaik-Eltern aus demselben Stamm. Wahrscheinlich sterben Eier, Embryonen und Larven mit bestimmten unregelmäßigen Chromosomenkombinationen in verschiedenen Stadien ab.

\section{BERICHTE}

\section{Arbeiten von L. Pauling und Mitarbeitern ${ }^{1}$ über die Bildung von Antikörpern in vitro und über Haptene mit 2 und mehr Haftgruppen}

In Jahre 1940 veröffentlichte L. P a u ling ${ }^{1 a}$ eine Theorie der Struktur und der Bildung von Antikörpern, die durch Arbeiten der Folgezeit eine weitgehende experimentelle Bestätigung erfahren hat. Die Theorie enthält zwei voneinander unabhängige Bestandteile: I. die Annahme, daß Antikörper aus normalen Serumeiweißstoffen durch eine Entfaltung und Neufaltung der Polypeptidketten hervorgehen; II. die Annahme, daß ein Antikörpermolekül 2 spezifische Haftgruppen hat, mit denen es sich an 2 Antigenmoleküle anzuheften vermag. I. steht im Zusammenhang mit der früher von Mirsky und Pa uling (Proc. Nat. Acad. Sci. USA. 22, 439 [1936]) aufgestellten Theorie der Denaturierung der Eiweißstoffe. Ein nativer Eiweißstoff ist danach ausgezeichnet durch eine ganz bestimmte, bei allen Molekülen in derselben Weise festgelegte Anordnung der Polypeptidkette oder Polypeptidketten, aus denen er besteht. Bei der Denaturierung werden lockere Bindungen zwischen den Ketten gelöst und neue wieder eingegangen, wofür eine Unzahl verschiedener Möglichkeiten bestehen, so daß die ursprünglich gleichartigen Moleküle untereinander ungleich werden. Diese Auffassung der Denaturierung wird u. a. gestiitzt durch die thermodynamische Auswertung reversibler Denaturierungsvorgänge. Es geht daraus hervor, daß die Entropie ron denaturiertem Eiweiß größer ist als von nativem Eiweiß. Da die Molekülgröße in den untersuchten Fällen der reversibeln Denaturierung ungeändert bleibt, so folgt daraus nach dem Satz von Boltzmann eine größere Zahl von Konfigurationsmöglichkeiten für ein denaturiertes Eiweißmolekül als für ein natives. In bezug auf die Bildung der Antikörper äußert P a u ling den Gedanken, daß in Gegenwart von Antigen und bei denaturierenden Einwirkungen auf die Serumglobuline eine Umfaltung der Moleküle derart erfolgen kann, daß das umgefaltete Molekül eine maximale Zahl von Nebenvalenzen gegen das Antigen-Molekül abzusättigen vermag, wodurch eine spezifische Bindung ermöglicht wird. In den Arbeiten b) und c) wird angegeben, daß es möglich ist, in vitro aus Serumglobulinen und auch aus Serumalbuminen Antikörper zu erzeugen, die spezifisch mit dem Antigen reagieren. Z. B. wurde der Azofarbstoff 1.3-Dioxy-2.4.6-tris[ $p$-azo-phenylarsensäure]-benzol bei $57^{\circ} 14$ Tage mit $\gamma$-Globulin vom Rind (hergestellt nach der Methode ron Cohn (J. Amer. chem. Soc. 62, 3396 [1940]) im Brutschrank gehalten. Aus dem Komplex von Azofarbstoff und Globulin konnte mit dem Hapten Arsanilsäure der Farbstoff verdrängt werden und durch Dialyse der Farbstoff und das Hapten rom Globulin abgetrennt werden. Das derartig behandelte $\gamma$-Globulin hatte die Fähigkeit gewonnen. spezifisch 